\title{
Correlation of clinical illness with viremia in Zika virus disease during an outbreak in Singapore
}

\author{
Deborah H. L. Ng${ }^{1}$, Hanley J. Ho², Angela Choww,3,4, Joshua Wong ${ }^{2}$, Win Mar Kyaw², Adriana Tan², Po Ying Chia', \\ Chiaw Yee Choy', Glorijoy Tan', Tsin Wen Yeo ${ }^{1,4}$ and Yee Sin Leo ${ }^{1,3,4}$
}

\begin{abstract}
Background: The first autochthonous Zika virus (ZIKV) outbreak in Singapore was detected in August 2016. We report an analysis of the correlation of clinical illness with viremia and laboratory parameters in this Asian cohort.

Methods: We conducted a prospective longitudinal cohort study of patients with a positive blood ZIKV polymerase chain reaction (PCR) result who were admitted to Tan Tock Seng Hospital, Singapore, for isolation and management.

Results: We included 40 patients in our study. Rash was present in all patients, while 80\% (32/40) had fever, 62.5\% (25/40) myalgia, 60\% (24/40) conjunctivitis and 38\% (15/40) arthralgia. The median duration of viremia was 3.5 days (IQR: 3-5 days). Patients with viremia of $\geq 4$ days were more likely to have prolonged fever compared to those with viremia of less than 4 days (95\% versus $63 \%, p=0.01$ ), but had no significant correlation with other clinical signs and symptoms, or laboratory investigations. However, 21 patients (53\%) had hypokalemia despite the absence of gastrointestinal symptoms.

Conclusion: Although fever correlated with duration of viremia, 30\% of patients remained viremic despite defervescence. Laboratory abnormalities such as leukopenia or thrombocytopenia were not prominent in this cohort but about half the patients were noted to have hypokalemia.
\end{abstract}

Keywords: Viremia, Zika, Outbreak, Singapore

\section{Background}

Zika virus (ZIKV), a mosquito-borne flavivirus, gained international recognition following reports of microcephaly and Guillain-Barré syndrome (GBS) associated with the disease in South America, which had prompted the World Health Organisation (WHO) to declare it a Public Health Emergency of International Concern on February 1, 2016 [1]. However, the presence of ZIKV has been recognised in Southeast Asia for over half a century.

\footnotetext{
* Correspondence: hanleyho@gmail.com

This work was presented as an abstract at the 1st joint meeting of the ISAAR (International Symposium on Antimicrobial Agents and Resistance) \& ICIC (International Interscience Conference on Infection and Chemotherapy) in Septemeber 2017.

${ }^{2}$ Department of Clinical Epidemiology, Office of Clinical Epidemiology, Analytics, and Knowledge, Tan Tock Seng Hospital, 11 Jalan Tan Tock Seng, Singapore 308433, Singapore

Full list of author information is available at the end of the article
}

Previous studies demonstrated serological evidence of ZIKV infections in Malaysia in 1969, and the first clinical infections were reported in Indonesia in 1977 [2].

On August 27, 2016, the first case of locally transmitted ZIKV disease in Singapore was identified. The patient was a 47-year-old female who had not travelled out of the country in the 2 weeks preceding her illness onset [3]. Active case finding by the Singapore Ministry of Health $(\mathrm{MOH})$ revealed further laboratory-confirmed infections, signalling the presence of a large community outbreak of ZIKV. A total of 455 cases were reported up until 30 November 2016 [4]. During the initial outbreak containment phase, all suspected ZIKV infections were screened at Tan Tock Seng Hospital (TTSH), an acute tertiary care general hospital, for further management. Isolation at the Communicable Disease Center (CDC) was mandated by $\mathrm{MOH}$ for all cases with a positive

(c) The Author(s). 2018 Open Access This article is distributed under the terms of the Creative Commons Attribution 4.0 International License (http://creativecommons.org/licenses/by/4.0/), which permits unrestricted use, distribution, and 
blood ZIKV polymerase chain testing (PCR) result, with daily testing until results were negative before discharging them back to the community.

ZIKV generally causes a mild febrile illness, characterised by symptoms of rash, arthralgia, non-purulent conjunctivitis and/or low-grade fever [5]. Viremia in ZIKV disease has been reported to range from the day of onset of symptoms for up to 11 days from symptom onset [6]. However, little is known about the correlation between clinical symptoms and signs, as well as laboratory parameters, with viremia. Whole genome sequencing of the virus demonstrated that the ZIKV strain circulating in Singapore was genetically distinct from the strain imported from Brazil in May 2016 [4]. This study therefore aims to describe the clinical characteristics of ZIKV infection and its correlation to viremia and other laboratory investigations, as observed during the ZIKV outbreak in Singapore. As clinical symptoms such as fever are known to correlate with viremia for other arbovirus infections such as dengue, we hypothesised that a similar correlation might exist for ZIKV infection.

\section{Methods}

We conducted a prospective longitudinal cohort study of patients with laboratory-confirmed ZIKV disease who were admitted to the CDC, TTSH from 26 August 2016 to 5 September 2016, when Singapore was in the containment phase of the ZIKV outbreak. TTSH is a 1500-bed teaching hospital, which also encompasses the CDC, the designated institution for centralised management of emerging infectious disease outbreaks in Singapore.

Active case finding for persons with suspected ZIKV disease was initiated by the $\mathrm{MOH}$ through public education and contact tracing. Adults living or working in the vicinity of the initial outbreak area with symptoms compatible with suspected ZIKV disease were advised to present directly to TTSH or were referred by primary care practitioners for further testing. The case definition for suspected ZIKV disease was any person with fever and maculopapular rash, plus one additional symptom of arthralgia, myalgia, headache or non-purulent conjunctivitis.

All suspect cases who fully or partially fulfilled the case definition had blood and urine samples tested for ZIKV ribonucleic acid (RNA) at Tan Tock Seng Hospital, using real-time reverse transcriptase polymerase chain reaction (rRT-PCR) methods as described by Lanciotti et al. [6] Blood and urine samples from the same specimens were also sent to the National Public Health Laboratory under the $\mathrm{MOH}$, also for concurrent testing using rRT-PCR, for concordance. During the containment phase, patients with a positive blood ZIKV PCR test were admitted to TTSH for clinical management and isolation to reduce the risk of community transmission, while those who only had a positive urine ZIKV
PCR test were discharged. All inpatients underwent daily blood ZIKV PCR testing and were discharged only when their blood tested negative for ZIKV.

Our study evaluated patients who had a positive blood sample for ZIKV by rRT-PCR testing, and correlated their period of viremia with their clinical and laboratory characteristics. Within this group of patients, we excluded those with discordant blood ZIKV PCR results between the two testing laboratories, those who had concurrent bacterial or viral infections on admission, and those who did not have a documented negative blood ZIKV PCR prior to discharge. Only blood samples that tested positive from both laboratories were included in the analysis to improve diagnostic accuracy as the testing platform was relatively new. Serial testing of urine for ZIKV PCR was not performed as it was used only at the point of presentation to confirm the diagnosis.

Clinical data, daily symptoms and signs and laboratory results for all patients were obtained from electronic medical records. A standardised ZIKV disease clinical care pathway was used for recording daily signs and symptoms, vital signs and management of all hospitalised patients. Clinical data collected included demographic information, presence of medical co-morbidities, travel history and contact history. Baseline investigations for all confirmed cases of ZIKV disease included a full blood count, renal function, liver function and dengue serology. Further investigations such as blood cultures and chest X-ray were performed if clinically indicated. The reference values for the normal range of laboratory tests were in accordance with those used by the hospital's laboratory. Duration of viremia was calculated from the day of onset of symptoms until the first negative blood ZIKV PCR result. Duration of clinical illness was calculated from the first day of onset of symptoms to the day of resolution of all symptoms.

Key laboratory parameters were visualised with box plots to investigate the overall trend over the course of ZIKV infection. Chi-square and Fisher's exact tests were used to evaluate differences in proportions for categorical variables, while Mann-Whitney $U$ test was used to evaluate differences in medians for continuous variables. A $p$ value of $<0.05$ was taken to be statistically significant. All statistical analyses were performed using Stata version 13 (StataCorp 2013, College Station, TX).

\section{Results}

A total of 359 patients were referred to the CDC for suspected ZIKV disease from 26 August to 5 September 2016. (Fig. 1) Of these, 72 patients yielded a positive blood ZIKV PCR test at the time of presentation, and were admitted to the CDC for isolation. Twenty patients were excluded from the analysis due to differences in blood results between the two laboratories. Another nine 


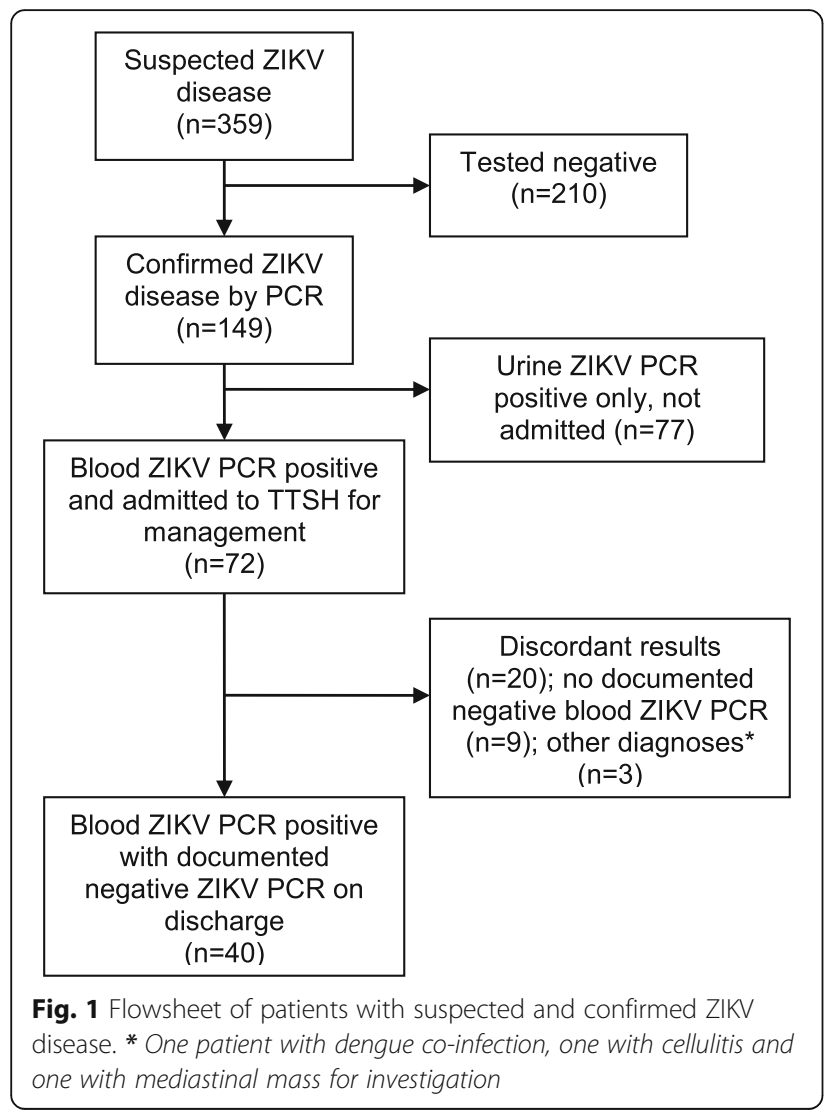

were excluded as they had no available negative blood ZIKV PCR result to indicate the end of viremia: five were discharged at the end of the containment phase with no negative blood PCR result available, and four patients had been transferred to another hospital. Of the remaining 43 patients, a further three were excluded as one had dengue co-infection, one had cellulitis, while the remaining patient had been admitted for investigation of a mediastinal mass and was incidentally found to have ZIKV infection.

Of the 40 inpatients, 24 (60\%) were male and 31 (76\%) were of Chinese ethnicity. Thirty-four (85\%) were Singaporeans while the remainder were foreigners residing in Singapore. The median age was 34 years [inter-quartile range (IQR) 26 to 52.5 years]. Five of them reported travel in the last 14 days prior to admission, all to Malaysia. None of the female patients was pregnant.

\section{Clinical presentation and correlation with viremia}

Among the 40 viremic patients, the median duration of viremia was 3.5 days (IQR: 3 to 5 days), while the median duration of symptoms was 3 days (IQR: 2 to 4 days). Table 1 documents the daily progression of combined signs and symptoms from onset of illness to the end of viremia.
Of the signs and symptoms, rash was the most common, being present in all patients. (Table 2) The rash was noted to be generalised and maculopapular in 31 of 40 patients $(79 \%)$ but distribution and character were unknown in 9 patients. The next most common sign and symptom was fever, present in 32 patients $(80 \%)$; followed by myalgia in 25 patients (62.5\%), conjunctivitis in 24 patients $(60 \%)$ and arthralgia in 15 patients (38\%).

The duration of symptoms did not exceed the duration of viremia in $32(80 \%)$ patients. Eight patients had symptoms beyond the duration of viremia, for which conjunctivitis was the predominant persisting symptom. Of these 8 patients, fever exceeded the duration of viremia only in one patient (subject 4). Of the 32 patients who had reported or documented fever, the end of viremia corresponded with defervescence in 17 patients (53\%). However, in 12 patients (30\%), viremia persisted for 1 day beyond the resolution of symptoms.

Vital signs were all within normal parameters, with no patients having tachypnea (respiratory rate $>28$ breaths per minute), tachycardia (pulse rate $>100$ beats per minute) or hypotension (systolic blood pressure $<90 \mathrm{mmHg}$ ). None of the patients had postural hypotension. All patients remained well during the course of illness with good recovery and none developed bleeding or neurological symptoms. There were no admissions to the intensive care unit and none of the patients was readmitted in the 3 months following their illness.

We divided the cohort into two groups based on duration of viremia, using a cut-off of 4 or more days to denote prolonged viremia. A higher proportion of patients with reported fever on the first day of illness was observed among patients with prolonged viremia (95\%), compared to those without prolonged viremia (63\%). There were otherwise no significant differences in symptoms and signs between the two groups. (Table 2) There was also no association between duration of viremia and the number of initial symptoms and signs $(p=0.26)$.

Laboratory results generally remained normal in this cohort, regardless of the duration of viremia. Leukopenia was not common, with only 17 patients (24\%) having a white cell count $<4.0 \times 10^{9} / \mathrm{L}$ and the lowest being $2.6 \times$ $10^{9} /$ L. Only 3 patients had thrombocytopenia (platelet count $<100 \times 10^{9} / \mathrm{L}$ ), the lowest being $83 \times 10^{9} / \mathrm{L}$. None of the patients showed significant hemoconcentration. Interestingly, 21 patients had hypokalemia (potassium level< $3.5 \mathrm{mmol} / \mathrm{L}$ ) despite the absence of anorexia or gastrointestinal symptoms. Of these, five patients had moderate hypokalemia (3.0-3.5 mmol/L), while one patient had severe hypokalemia $(2.3 \mathrm{mmol} / \mathrm{L})$. Only 5 patients had a creatinine of more than $105 \mathrm{umol} / \mathrm{L}$, but none of the readings were more than 1.5 times their baseline (if known). Two of them had mild hypokalemia. Only one patient had an elevated aspartate aminotransferase (AST) 
Table 1 Daily trend of symptoms and signs with viremia in 40 patients with ZIKV disease

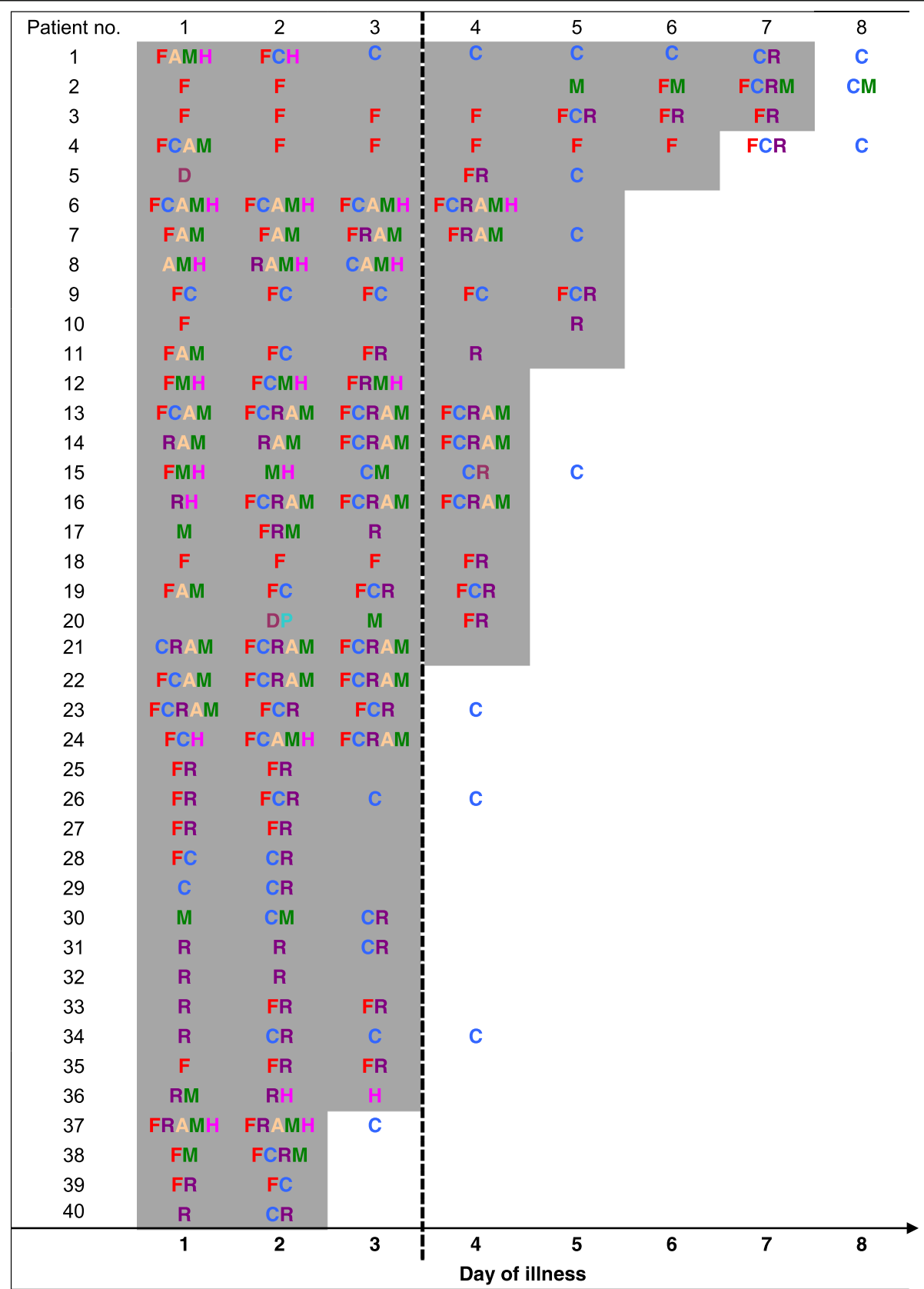

Positive ZIKV PCR.

Negative ZIKV PCR.

$F$ fever, $C$ conjunctivitis, $R$ rash, $H$ headache, $A$ arthralgia, $M$ myalgia, $D$ diarrhea, $P$ abdominal pain.

of $174 \mathrm{U} / \mathrm{L}$ and alanine aminotransferase (ALT) of $253 \mathrm{U} /$ $\mathrm{L}$ but further workup showed that the patient subsequently tested positive for Hepatitis C. Analysis of the laboratory investigations between the two groups also showed that there were no significant differences in values.

Box plots of the white cell count, lymphocyte count, hematocrit and ALT plotted by day of illness also showed that there were no significant differences in laboratory values for each day of illness. (Fig. 2).

\section{Discussion}

In our cohort of blood ZIKV PCR positive patients, clinical presentation was characterised by symptoms and signs of rash, fever, myalgia, arthralgia and non-purulent conjunctivitis, with only a small proportion reporting 
Table 2 Correlation of symptoms, signs and laboratory investigations on admission with viremia

\begin{tabular}{|c|c|c|c|}
\hline Symptoms and signs on admission & $\begin{array}{l}\text { Viremia }<4 \text { days } \\
(n=19) \\
n_{1}(\%)\end{array}$ & $\begin{array}{l}\text { Viremia } \geq 4 \text { days } \\
(n=21) \\
n,(\%)\end{array}$ & $p$-value \\
\hline Fever $^{a}$ & $12(63 \%)$ & $20(95 \%)$ & 0.01 \\
\hline Conjunctivitis $^{a}$ & $10(53 \%)$ & $14(67 \%)$ & NS \\
\hline Rash $^{a}$ & $19(100 \%)$ & $21(100 \%)$ & NS \\
\hline Arthralgia & $5(26 \%)$ & $10(48 \%)$ & NS \\
\hline Myalgia & $9(47 \%)$ & $16(76 \%)$ & NS \\
\hline Headache & $4(21 \%)$ & $6(29 \%)$ & NS \\
\hline Diarrhea & $3(16 \%)$ & $3(14 \%)$ & NS \\
\hline Abdominal pain & $0(0 \%)$ & $1(5 \%)$ & NS \\
\hline Nausea/ vomiting & $0(0 \%)$ & $1(5 \%)$ & NS \\
\hline Disorientation & $0(0 \%)$ & $0(0 \%)$ & NS \\
\hline Jaundice & $0(0 \%)$ & $0(0 \%)$ & NS \\
\hline Lymphadenopathy & $1(5 \%)$ & $1(5 \%)$ & NS \\
\hline Pharyngitis & $1(5 \%)$ & $3(14 \%)$ & NS \\
\hline Abnormal lung findings: crackles/ wheeze & $0(0 \%)$ & $0(0 \%)$ & NS \\
\hline \multicolumn{4}{|l|}{ Laboratory investigations (median, IQR) } \\
\hline White cell count, $10^{9} / \mathrm{L}$ & $3.6(3-10.3)$ & $5.3(2.7-6.5)$ & NS \\
\hline Hemoglobin, g/dL & $13.6(10.5-17.9)$ & $14.8(12.6-16.4)$ & NS \\
\hline Platelet count, $10^{9} / \mathrm{L}$ & $176(117-339)$ & $188(99-291)$ & NS \\
\hline Hematocrit, \% & $41.5(33.9-52.5)$ & $45(36-51.1)$ & NS \\
\hline Potassium, mmol/L & $3.45(3.2-3.8)$ & $3.65(2.3-3.9)$ & NS \\
\hline Urea, mmol/L & $3.4(2.4-4.9)$ & $2.1(1.9-3.4)$ & NS \\
\hline Creatinine, unit & $68(47-95)$ & $60(46-112)$ & NS \\
\hline Neutrophils & $2.17(1.73-4.67)$ & $3.21(1.06-4.66)$ & NS \\
\hline Lymphocytes & $1.14(0.63-4.28)$ & $1.12(0.58-1.72)$ & NS \\
\hline AST, IU & $26(18-40)$ & $30(17-124)$ & NS \\
\hline ALT, IU & $20(14-71)$ & $22(15-196)$ & NS \\
\hline Bilirubin & $12(8-13)$ & $11(9-19)$ & NS \\
\hline Albumin, $g / L$ & $39(37-46)$ & $39(32-43)$ & NS \\
\hline Number of initial symptoms and signs & $2(1-2)$ & $3(1-3)$ & NS \\
\hline
\end{tabular}

${ }^{a}$ denotes combined signs and symptoms

gastrointestinal symptoms such as diarrhea, abdominal pain, nausea and/or vomiting. This was similar to a study of 57 patients with ZIKV disease in Brazil, which also reported rash as the most common symptom, followed by headache, fever, arthralgia and myalgia [7].

We observed that $63 \%$ of patients reported or were documented to have fever on the first day of illness, suggesting that this could be the start of viremia (Table 1). In addition, fever showed better correlation with the period of viremia than other symptoms such as rash, myalgia and conjunctivitis. Nevertheless, a sizeable proportion of patients had persistent viremia even after fever had resolved. Other symptoms such as rash (the most commonly reported symptom) and conjunctivitis tended to appear only toward the middle or end of the illness. In addition, over a third of patients were no longer symptomatic while remaining viremic. Our findings differ from that of dengue, another flavivirus, where viremia has been reported to correlate with defervescence [8], and suggest that the neither the duration of any symptoms of ZIKV, nor the total number of symptoms on presentation, correlate well with the duration of viremia. While patients are often counselled to practise safe sex, owing to reports of sexual transmission of ZIKV [9], our findings suggest the possibility of continued transmission to the Aedes mosquito vector even after resolution of symptoms and signs. Hence, they support the continued use of personal protective measures to prevent mosquito bites and mosquito control interventions and the continuation of environmental public 


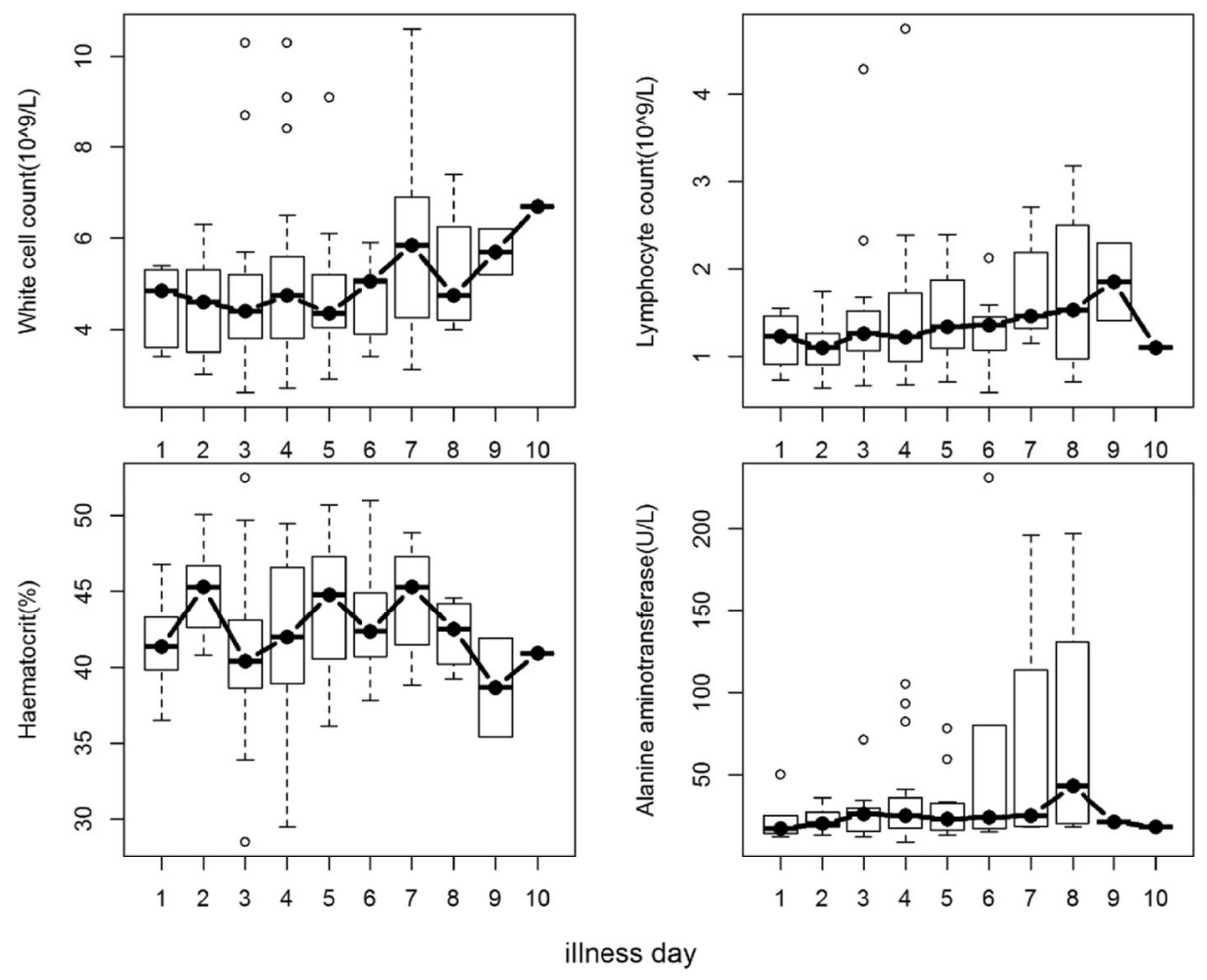

Fig. 2 Box plots of laboratory markers by day of illness

health measures to reduce the mosquito population to reduce transmission risk.

Previous studies have reported laboratory abnormalities such as leukopenia, neutropenia, thrombocytopenia or deranged liver function tests [10]. However, findings of our study demonstrated that these abnormalities are generally uncommon, with none of the patients with ZIKV disease having abnormal liver function tests. In addition, there were no significant variations in laboratory values across each day of illness. Interestingly, however, half our patients were found to have hypokalemia despite the absence of anorexia or symptoms to suggest gastrointestinal loss. Dengue fever has been reported to cause proteinuria or glomerulonephritis [11]. Similarly, the presence of ZIKV in the urine suggests that there is a possibility of the virus also causing glomerular injury, resulting in renal potassium loss. An in vitro study of renal cells infected with ZIKV showed that there were high levels of viral replication within different renal cell types, especially podocytes, the damage to which could initiate progressive renal impairment [12]. In our cohort, none of the patients had evidence of renal impairment. However, we were unable to conduct detailed studies of renal function or trend the results. Further studies are required to correlate the level of viruria with the degree of renal impairment and hypokalaemia. Nonetheless, from a clinical perspective, it may be prudent to also check potassium levels in patients diagnosed with ZIKV disease.

A strength of this paper is the well-characterised Asian cohort, with daily symptoms and signs clearly documented according to a standardised clinical care pathway. Limitations of the study include the small size of the cohort, and the subjectivity of symptoms and possible under-reporting by patients. It was therefore difficult to ascertain if the duration and level of viremia had any impact on development of future complications, such as GBS.

\section{Conclusions}

This is the first known paper examining the correlation of symptoms and signs of viremia in ZIKV disease in an Asian cohort. Our study demonstrated that the duration of ZIKV viremia did not correlate well with the progression of clinical disease or symptoms, unlike that seen in that of dengue fever. In addition, a sizeable proportion of patients may still be viremic despite resolution of symptoms and signs. While laboratory abnormalities were not a prominent feature of ZIKV disease, over half the patients had hypokalemia, which may be suggestive of direct glomerular injury by the virus, although further pathological studies are required to confirm this. 


\section{Abbreviations}

ALT: alanine aminotransferase; AST: aspartate aminotransferase; CDC: Communicable Disease Center; GBS: Guillain-Barré syndrome; $\mathrm{MOH}$ : Ministry of Health; PCR: polymerase chain reaction; RNA: ribonucleic acid; rRT-PCR: Real-time reverse transcriptase polymerase chain reaction; TTSH: Tan Tock Seng Hospital; WHO: World Health Organisation; ZIKV: Zika virus

\section{Availability of data and materials}

The datasets supporting the conclusions of this article are included within the article and its additional files.

\section{Authors' contributions}

AC, TY and YL conceived the presented idea. DN, HH, WMK, AT, PYC, CYC, and GSET collected the data. AT, JW and $\mathrm{HH}$ performed the data analysis. DN, PYC, CYC, GSET, JW and HH wrote the manuscript with support from AC and $Y L$. All authors read and approved the final manuscript.

\section{Ethics approval and consent to participate}

The study was approved by the Domain Specific Review Board of the National Healthcare Group, Singapore, under which the institution belonged (NHG DSRB Reference No: 2016/01027)

\section{Consent for publication}

Not applicable.

\section{Competing interests}

Angela Chow is an Associate Editor for BMC Infectious Diseases. All other authors declare that they have no competing interests.

\section{Publisher's Note}

Springer Nature remains neutral with regard to jurisdictional claims in published maps and institutional affiliations.

\section{Author details}

National Centre for Infectious Diseases, Moulmein Road, Singapore 308433, Singapore. ${ }^{2}$ Department of Clinical Epidemiology, Office of Clinical Epidemiology, Analytics, and Knowledge, Tan Tock Seng Hospital, 11 Jalan Tan Tock Seng, Singapore 308433, Singapore. ${ }^{3}$ Saw Swee Hock School of Public Health, National University of Singapore, 12 Science Drive 2, Singapore 117549, Singapore. ${ }^{4}$ Lee Kong Chian School of Medicine, Nanyang

Technological University, 11 Mandalay Road, Singapore 308232, Singapore.

Received: 22 January 2018 Accepted: 26 June 2018

Published online: 04 July 2018

\section{References}

1. World Health Organisation. WHO Director-General summarizes the outcome of the Emergency Committee regarding clusters of microcephaly and Guillain-Barré syndrome. http://www.who.int/ mediacentre/news/statements/2016/emergency-committee-zikamicrocephaly/en/. Accessed 14th Sept 2016.

2. Musso D, Gubler DJ. Zika virus. Clin Microbiol Rev. 2016;29:487-524

3. Leo Y, Chow A. Zika virus has arrived in Singapore. Lancet Inf Dis. 2016; 16(12):1317-9.

4. The Singapore Zika Study Group. Outbreak of Zika virus infection in Singapore: an epidemiological, entomological, virological, and clinical analysis. Lancet Inf Dis. 2017;17(8):813-21.

5. Duffy MR, Chen T-H, Hancock WT, Powers AM, Kool JL, Lanciotti RS, et al. Zika virus outbreak on Yap Island, Federated States of Micronesia. N Engl J Med. 2009;360:2536-43.

6. Lanciotti RS, Kosoy OL, Laven JJ, Velez JO, Lambert AJ, Johnson AJ, et al. Genetic and serologic properties of Zika virus associated with an epidemic, yap state, Micronesia, 2007. Emerg Inf Dis. 2008;14(8):1232-9. https://doi.org/ 10.3201/eid1408.080287.

7. Cerbino-Neto J, Mesquita EC, Souza TM, Parreira V, Wittlin BB, Durovni $B$, et al. Clinical manifestations of Zika virus infection, Rio de Janeiro, Brazil. 2015 Emerg Infect Dis. 2016;22(7):1318-20. https://doi.org/10. 3201/eid2207.160375
8. Vaughn DW, Green S, Kalayanarooj S, Innis BL, Nimmanitya S, Suntayakorn S, et al. Denque in the early febrile phase: viremia and antibody responses. J Infect Dis. 1997;176:322-30.

9. D'Ortenzio E, Matheron S, Yazdanpanah Y, de Lamballerie X, Hubert B, Piorkowski G, et al. Evidence of sexual transmission of Zika virus. N Engl J Med. 2016;374(22):2195-8.

10. Plourde AR, Bloch EM. A literature review of Zika virus. Emerg Infect Dis. 2016;22(7):1185-92.

11. Lizarraga KJ, Nayer A. Dengue-associated kidney disease. J Nephropathol. 2014;3(2):57-62. https://doi.org/10.12860/jnp.2014.13. Epub 2013 Dec 28

12. Alcendor DJ. Zika virus infection of the human glomerular cells: implications for viral reservoirs and renal pathogenesis. J Infect Dis. 2017;216(2):162-71. https://doi.org/10.1093/infdis/jix171.

\section{Ready to submit your research? Choose BMC and benefit from:}

- fast, convenient online submission

- thorough peer review by experienced researchers in your field

- rapid publication on acceptance

- support for research data, including large and complex data types

- gold Open Access which fosters wider collaboration and increased citations

- maximum visibility for your research: over $100 \mathrm{M}$ website views per year

At BMC, research is always in progress.

Learn more biomedcentral.com/submissions 